\title{
Biodiversité fonctionnelle en paysage agricole : étude floristique et syrphidologique de Surfaces de Promotion de la Biodiversité (SPB)
}

\author{
Marie Bessat ${ }^{(1,2)}$, Emmanuel Castella ${ }^{(2)}$, Martin C.D. Speight ${ }^{(3)}$, Dominique Fleury ${ }^{(3)}$, \\ Nicolas Delabays ${ }^{(4,5)}$
}

(1) République et Canton de Genève. Département du territoire (DT). Office cantonal de l'Agriculture et de la Nature (OCAN). Rue des Battoirs, 7.CH-1205 Genève (Suisse).E-mail : mariebessat@ gmail.com

(2) Département F.A. Forel des Sciences de l'Environnement et de l'Eau \& Institut des Sciences de l'Environnement. Université de Genève. Boulevard Carl-Vogt, 66. CH-1206 Genève (Suisse).

${ }^{(3)}$ Department of Zoology. Trinity College. Dublin 2 (Ireland).

(4) République et Canton de Genève. Département du territoire (DT). Office cantonal de l'Agriculture et de la Nature (OCAN). Service de l'Agronomie (SAgr). Chemin Pont-du-Centenaire, 109. CH-1228 Plan-les-Ouates (Suisse).

${ }^{(5)}$ Hepia. HES-SO/Genève. Institut Terre Nature Environnement. Route de Presinge, 150. CH-1254 Jussy (Suisse).

Reçu le 4 février 2019, accepté le 3 septembre 2019, mis en ligne le 5 novembre 2019.

Cet article est distribué suivant les termes et les conditions de la licence CC-BY (http://creativecommons.org/licenses/by/4.0/ deed.fr)

Description du sujet. Cette étude traite de la végétation et des Diptères Syrphidae au sein de Surfaces de Promotion de la Biodiversité (SPB) de type «prairies extensives » en contexte agricole.

Objectifs. L'objectif consiste à tester l'influence des facteurs « taille » et « qualité botanique » des SPB sur la syrphifaune qui s'y développe. Il s'agit également de rechercher les relations entre la végétation et les syrphes, ainsi que de mettre en avant le potentiel de lutte biologique de ces surfaces.

Méthode. Douze SPB ont été sélectionnées, sur la base de leur taille $\left(<3000 \mathrm{~m}^{2} v s>9000 \mathrm{~m}^{2}\right)$ et de leur niveau de qualité botanique établi sur la base de la présence d'espèces végétales indicatrices. Des inventaires de végétation ont été réalisés dans chacune des SPB, l'échantillonnage des syrphes a été effectué par la pose de deux pièges à émergence et d'un piège Malaise par site.

Résultats. Indépendamment de leur taille ou de leur niveau de qualité botanique, les SPB abritent et attirent une faune syrphidologique majoritairement aphidiphage au stade larvaire. Les fleurs de couleur jaune et blanche ainsi que celles produisant du nectar influencent la richesse et l'abondance en syrphes.

Conclusions. Le potentiel des SPB à abriter et attirer des syrphes aphidiphages montre qu'elles sont en mesure d'offrir un service de lutte biologique contre les ravageurs des cultures adjacentes.

Mots-clés. Lutte biologique, habitats, végétation, Diptères Syrphidae, Syrph-the-Net, canton de Genève, Suisse .

Functional biodiversity in an agricultural landscape: a floristic and syrphidologic study of Biodiversity Promotion Surfaces (BPSs)

Description of the subject. This study focuses on the vegetation and Diptera Syrphidae found in biodiversity promotion surfaces (BPSs) of the "extensive meadows" type, within an agricultural context.

Objectives. The objective was to test the influence of the "size" and "botanical quality" factors of BPSs on the syrphifauna that develops there. It also aimed to highlight the relationships between vegetation and hoverflies, and to emphasize the potential for the biological control of these surfaces.

Method. Twelve BPSs were selected on the basis of their size $\left(<3,000 \mathrm{~m}^{2} v s>9,000 \mathrm{~m}^{2}\right)$ and their level of botanical quality, defined on the basis of indicator plant species. Vegetation inventories were conducted in each of the BPSs. Hoverfly sampling was carried out via the two emergence traps and one Malaise trap that were installed on each site. 
Results. Regardless of their size or level of botanical quality, BPSs are home to and attract mostly aphidiphagous hoverflies in the larval stage. Yellow and white flowers, as well as those producing nectar, influence the richness and abundance of hoverflies.

Conclusions. The potential of BPSs to shelter and attract aphidiphagous hoverflies shows that they are able to offer a biological control service against pests in adjacent crops.

Keywords. Conservation biological control, vegetation, Diptera Syrphidae, Syrph-the-Net, Geneva canton, Switzerland.

\section{INTRODUCTION}

En Suisse, les Surfaces de Promotion de la Biodiversité (SPB) sont depuis 1993 considérées comme des externalités positives mises en place par la politique agricole fédérale, rémunérées dans le but de promouvoir la diversité biologique dans les agroécosystèmes (Caillet-Bois et al., 2016). Leur mise en place fait partie des prestations écologiques requises, un programme environnemental obligatoire. L'obtention de paiements directs nécessite la mise en place de SPB sur au moins $7 \%$ de la surface agricole utile $(3,5 \%$ pour les cultures spéciales comme la vigne). Les paiements directs octroyés aux agriculteurs varient en fonction du type de SPB (jachères, prairies extensives, haies, vergers, etc.), de leur qualité botanique ainsi que de leur intégration dans un réseau agro-environnemental (OCAN, 2019). Ces contributions écologiques ont participé à la reconstruction d'un paysage agricole hétérogène en implantant des habitats semi-naturels non cultivés qui peuvent être favorables aux auxiliaires des cultures (Landis et al., 2000). En 2018, l'ensemble des SPB du canton de Genève recouvraient plus de $14 \%$ de sa surface agricole utile (OCAN, 2019). Bien que le rôle de ces surfaces pour la biodiversité constitue un enjeu important, le rôle qu'elles jouent dans le maintien de la diversité fonctionnelle dans un paysage agricole en ce qui concerne le contrôle des ravageurs des cultures demeure encore peu étudié. De nombreuses études montrent le rôle écosystémique que jouent les habitats non cultivés naturels ou semi-naturels dans la matrice agricole (Östman et al., 2001 ; Gurr et al., 2004 ; Tscharntke et al., 2005); ils constituent des habitats alternatifs aux cultures, des stocks de ressources alimentaires, ainsi que des sites d'hivernation pour les auxiliaires qui n'effectuent généralement pas la totalité de leur cycle de vie dans les cultures (Ouvrard et al., 2018 ; Ganser et al., 2019). Contrairement à la lutte biologique classique, dans laquelle les ennemis naturels des ravageurs sont introduits dans les cultures, la lutte biologique par conservation vise l'attraction de ceux-ci par des modifications structurelles de l'environnement ou par le changement des pratiques (réduction des intrants comme les phytosanitaires, fauches tardives, etc.). Le but d'une telle lutte est la protection et/ou l'amélioration des populations d'auxiliaires afin de réduire les effets négatifs des ravageurs des cultures (Hajek, 2004).

Plusieurs caractéristiques des habitats seminaturels, comme l'arrangement spatial et le degré de connectivité des patchs ou leur nature (Thies et al., 2003; Tscharntke \& Brandl, 2004) influencent le potentiel biologique et fonctionnel de ces surfaces. La taille des habitats semi-naturels ainsi que leur qualité botanique sont deux facteurs, bien que très controversés, influençant leur diversité végétale et entomologique. Parmi les insectes, les Diptères Syrphidae sont des pollinisateurs actifs au stade adulte et possèdent des larves aphidiphages chez de nombreuses espèces. Ils font partie des auxiliaires contribuant à la lutte biologique contre les ravageurs des cultures et dépendent des habitats naturels et semi-naturels pour leur cycle de vie (Speight, 2015). Si certaines études mettent en avant une relation positive entre la taille du patch et sa diversité végétale ou syrphidologique (Ouin et al., 2006 ; Meyer et al., 2009), d'autres avancent que c'est plutôt la quantité de ressources en pollen et en nectar (Haenke, 2009) ou la qualité et la densité en fleurs des SPB (Albrecht et al., 2007) qui jouent un rôle prépondérant pour les populations de syrphes. Kleijn \& Van Langevelde (2006) relatent un effet positif des habitats semi-naturels sur l'abondance en syrphidés quand la couverture en fleurs augmente. Pour Cowgill et al. (1993), les syrphes sélectionnent les ressources florales en fonction de leur disponibilité. Par contre, Schaffers et al. (2008) concluent que c'est la diversité botanique qui constitue un facteur clé influençant les assemblages d'insectes, notamment la richesse spécifique des syrphidés (Burgio et al., 2015). À un niveau plus fin, la variation des préférences florales entre espèces de syrphes demeure imparfaitement connue. La couleur des fleurs (Lazaro et al., 2008), ainsi que leur morphologie qui conditionne notamment l'accessibilité au nectar et au pollen pour des insectes à pièces buccales courtes (Gilbert, 1981), sont des critères déterminants. Speight \& Castella (2015) mettent en relation les espèces de syrphes et certaines caractéristiques florales, en particulier la production de nectar.

La présente étude se focalise sur les SPB de type prairies extensives et sur deux caractéristiques ayant une influence potentielle sur leur biodiversité : 
- leur taille (petites SPB : $<3000 \mathrm{~m}^{2}$ vs grandes SPB : $\left.>9000 \mathrm{~m}^{2}\right)$;

- leur niveau de qualité requis pour l'homologation en Suisse (qualité inférieure QI vs qualité supérieure QII).

Les conditions pour que les prairies extensives soient inscrites en niveau de qualité I sont les suivantes: bandes refuges sur $10 \%$ de la surface totale, pas d'utilisation de fumure ni de produits phytosanitaires, fauche dès le 15 juin, broyage interdit, exportation de la récolte, utilisation de la SPB sur une durée d'au minimum huit ans sans interruption. Les SPB de qualité II doivent, en plus des conditions précédentes, abriter au minimum six espèces végétales indicatrices parmi une cinquantaine de taxons.

Les objectifs de l'étude sont de trois ordres :

- caractériser la syrphifaune des SPB en fonction des deux catégories de taille et des deux niveaux de qualité. Les critères permettant de distinguer les deux niveaux de qualité étant exclusivement botaniques, il s'agit de rechercher si cette distinction peut se répercuter sur les communautés de syrphes ;

- caractériser le potentiel en termes de lutte biologique de ces surfaces à travers la présence d'espèces de syrphes à larves aphidiphages ;

- rechercher les liens potentiels entre la végétation des SPB et la faune syrphidologique qui s'y développe au stade larvaire ainsi que celle les fréquentant au stade adulte. L'originalité de l'étude est en effet de coupler deux méthodes d'échantillonnage visant à caractériser simultanément la faune syrphidologique fréquentant les SPB au stade adulte et larvaire (faune émergente).

\section{MÉTHODES}

\subsection{Caractéristiques des sites d'étude}

Douze SPB de type «prairies extensives » ont été échantillonnées dans le canton de Genève (altitude variant de 410 à $480 \mathrm{~m}$ ) (Tableau 1). Elles ont été sélectionnées parmi les plus de 1000 SPB de ce type (930,96 ha) dans le canton de Genève (OCAN, 2019) afin de réduire au mieux la variabilité des conditions autres que leur taille et leur niveau de qualité. En particulier, les parcelles retenues s'inscrivent dans un contexte à dominante agricole où la proportion de formations boisées dans un rayon de $200 \mathrm{~m}$ a été minimisée. Elles font partie d'un réseau agroenvironnemental et leur taille ainsi que leur ratio périmètre-aire sont les plus homogènes possibles pour une même catégorie de taille. Les 12 sites
Tableau 1. Coordonnées géographiques (CH 1903+/LV95) et communes des 12 sites - Geographical coordinates (CH 1903+/LV95) and municipalities of the 12 sites.

\begin{tabular}{lll}
\hline Site & Commune & Coordonnées géographiques \\
\hline 1PQI & Choulex & $6,222907 / 46,225565$ \\
\hline 2PQI & Jussy & $6,256665 / 46,231637$ \\
3PQI & Jussy & $6,255742 / 46,236198$ \\
1PQII & Choulex & $6,230895 / 46,230412$ \\
\hline 2PQII & Jussy & $6,274183 / 46,240199$ \\
3PQII & Meinier & $6,234686 / 46,251307$ \\
1GQI & Dardagny & $5,994455 / 46,192513$ \\
\hline 2GQI & Russin & $6,018075 / 46,194882$ \\
3GQI & Dardagny & $6,000583 / 46,191049$ \\
\hline 1GQII & Dardagny & $5,998671 / 46,182454$ \\
2GQII & Jussy & $6,247704 / 46,233543$ \\
\hline 3GQII & Gy & $6,254907 / 46,249331$ \\
\hline
\end{tabular}

retenus ont finalement été sélectionnés sur la base de leur taille $\left(<3000 \mathrm{~m}^{2} v s>9000 \mathrm{~m}^{2}\right)$ et de leur qualité botanique (QI vs QII). Le croisement de ces deux critères conduit à quatre types de SPB représentés chacun par trois réplicats. Huit sites se situent à l'est du canton dans un rayon de $5 \mathrm{~km}$ et les quatre autres à l'ouest, dans un rayon de $3 \mathrm{~km}$ (Figure 1).

\subsection{Les bioindicateurs retenus}

La végétation est choisie car elle constitue le premier échelon de la chaine trophique et joue donc un rôle majeur pour les échelons supérieurs, comme les insectes ravageurs ou les auxiliaires (Zurbrügg \& Frank, 2006). C'est également la végétation qui sert de base à la désignation de la classe de qualité des SPB. Les Diptères Syrphidae ont été choisis pour évaluer le rôle des SPB en tant que surfaces hébergeant et attirant (zone de ponte et de vie larvaire et alimentation des insectes adultes) des insectes auxiliaires. Les Syrphidae sont considérés comme des indicateurs de la qualité entomologique des habitats naturels et semi-naturels pour des raisons développées par Sommaggio (1999) et Speight \& Castella (2001), parmi lesquelles figurent : leur rôle d'auxiliaires (pollinisation par les adultes, consommation de pucerons par certaines larves), l'existence d'une méthode d'échantillonnage simple et standardisable et l'ensemble des connaissances disponibles sur le groupe, rassemblées dans la base de données Syrph-the-Net (StN) (Speight \& Castella, 2015 ; Speight, 2015). StN renseigne en particulier sur les traits biologiques et écologiques des espèces et leur association à des catégories d'habitats. 


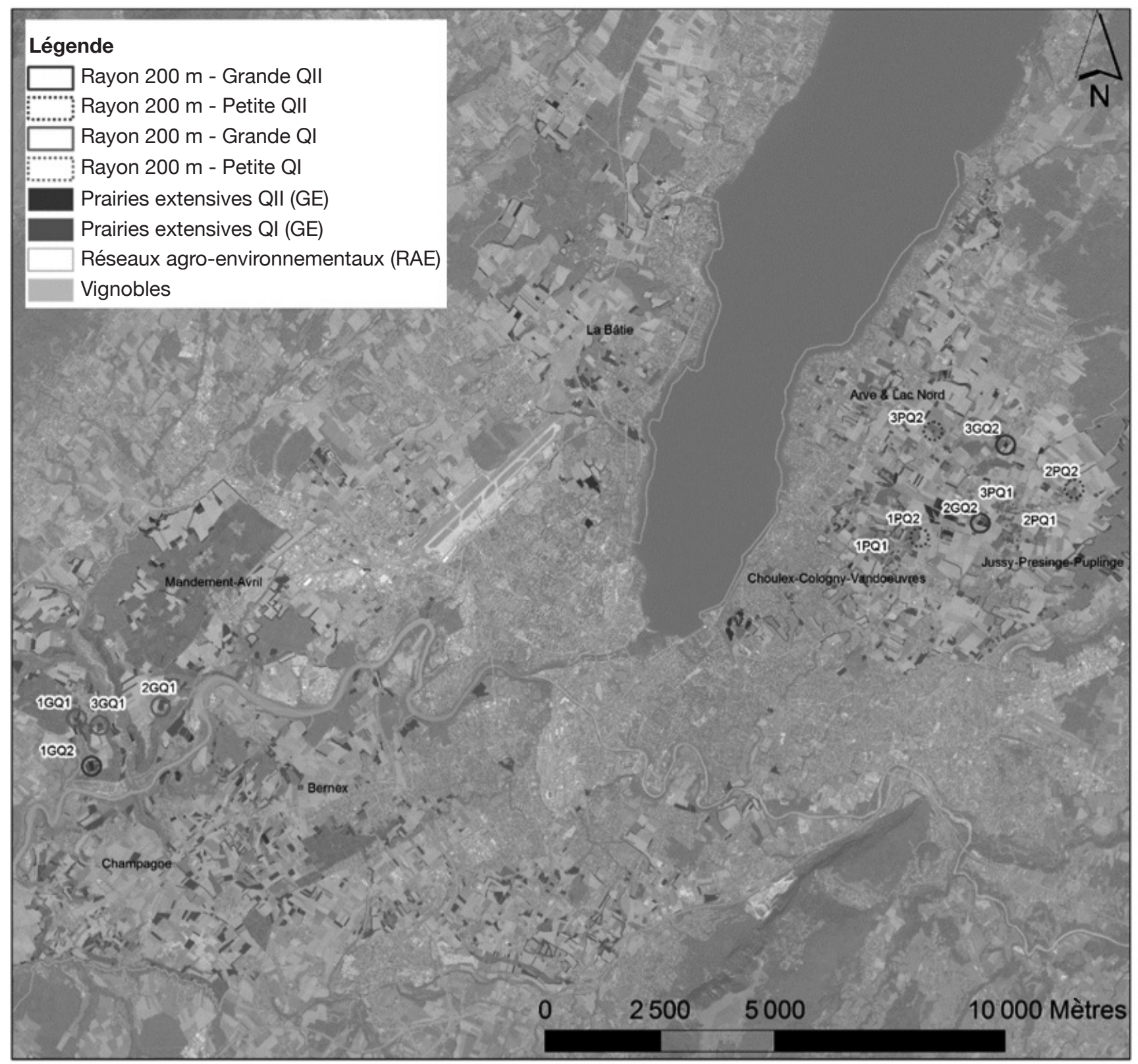

Figure 1. Carte générale positionnant l'emplacement des 12 sites retenus dans le canton de Genève - General map positioning the location of 12 sites in the canton of Geneva.

\subsection{Acquisition des données botaniques}

Quatre relevés de $1 \mathrm{~m}^{2}$ chacun (quadrats) ont été réalisés par type de Formation Végétale (FV : zones au sein de la SPB qui se distinguent par leur composition botanique, sur une surface représentative de la communauté végétale sélectionnée) dans le but d'obtenir une mesure comparative entre les différentes SPB, mais également de maximiser la richesse végétale inventoriée. Les quadrats ont été placés le plus au centre possible des FV (afin d'éviter les effets bordures) de sorte à former un carré de $6 \mathrm{~m}$ de côté ou un transect de $18 \mathrm{~m}$ de long dans les cas où les FV étaient trop étroites. Dans chaque quadrat, ont été relevées: la composition floristique, la structure de la végétation selon l'échelle de Londo (1976) (Tableau 2), ainsi que l'abondance des plantes en fleurs (Tableau 3). Afin d'obtenir un inventaire quasi-exhaustif de la richesse botanique de chaque SPB, les relevés de végétation ont été complétés par un inventaire complémentaire, consistant à parcourir l'ensemble de la SPB afin de recenser les taxons qui n'auraient pas été inventoriés dans les quadrats. Ce protocole a été répété fin avril et début juin 2017 (avant le 15 juin, date réglementaire de fauche pour l'octroi des subventions) (Bessat et al., 2018). 
Tableau 2. Recouvrement moyen des espèces végétales inventoriées dans les quadrats $\times 2$ dates $x$ 3SPB par catégorie de SPB (voir Tableau 1) - Average overlay of plant species inventoried in quadrats $x 2$ dates $x 3 S P B$ by category of BPS (see Table 1).

\begin{tabular}{|c|c|c|c|c|}
\hline \multirow{2}{*}{$\begin{array}{l}\text { Espèce végétale } \\
\text { (www.infoflora.ch/fr) }\end{array}$} & \multicolumn{4}{|c|}{ Recouvrement moyen (\%) } \\
\hline & PQI & PQII & GQI & GQII \\
\hline Achillea millefolium L. s.str. & 4,3 & 6,0 & 9,2 & 2,3 \\
\hline Agrimonia eupatoria L. & 0,0 & 0,0 & 0,8 & 2,4 \\
\hline Agrostis stolonifera $\mathrm{L}$. & 0,0 & 0,4 & 0,0 & 0,0 \\
\hline Ajuga reptans $\mathrm{L}$. & 0,0 & 0,0 & 2,2 & 0,3 \\
\hline Alopecurus pratensis L. & 0,0 & 0,0 & 0,2 & 0,0 \\
\hline Anthoxanthum odoratum $\mathrm{L}$. & 0,0 & 7,7 & 0,8 & 7,5 \\
\hline Arabidopsis thaliana (L.) Heynh. & 0,0 & 0,0 & 0,0 & 0,1 \\
\hline Arabis hirsuta (L.) Scop. & 0,0 & 0,3 & 0,0 & 0,0 \\
\hline Arenaria serpyllifolia aggr. & 0,7 & 0,0 & 0,0 & 1,6 \\
\hline Arrhenatherum elatius (L.) P.Beauv. ex J.Presl. \& C.Presl. & 47,5 & 15,0 & 15,8 & 3,7 \\
\hline Bellis perennis $\mathrm{L}$. & 1,6 & 2,3 & 0,2 & 0,0 \\
\hline Briza media $\mathrm{L}$. & 0,0 & 0,0 & 0,0 & 0,2 \\
\hline Bromus erectus Huds. s.str. & 0,0 & 24,3 & 0,0 & 35,2 \\
\hline Bromus hordeaceus L. & 1,7 & 0,2 & 0,7 & 1,4 \\
\hline Carex hirta L. & 0,5 & 0,0 & 0,0 & 0,0 \\
\hline Carex spicata Huds. & 0,0 & 0,0 & 0,5 & 0,0 \\
\hline Centaurea cyanus L. & 0,0 & 0,0 & 0,0 & 0,3 \\
\hline Centaurea jacea L. s.str. & 0,0 & 3,5 & 1,7 & 0,7 \\
\hline Centaurea scabiosa L. s.str. & 0,0 & 0,0 & 0,0 & 0,2 \\
\hline Cerastium arvense L. s.str. & 0,0 & 0,0 & 1,5 & 0,0 \\
\hline Cerastium fontanum subsp. vulgare (Hartm.) Greuter \& Burdet & 0,4 & 0,7 & 0,5 & 0,1 \\
\hline Cerastium glomeratum Thuill. & 0,0 & 0,0 & 0,0 & 0,6 \\
\hline Cichorium intybus L. & 0,0 & 0,0 & 2,5 & 0,0 \\
\hline Cirsium arvense (L.) Scop. & 0,0 & 0,5 & 0,2 & 0,6 \\
\hline Cirsium vulgare (Savi) Ten. & 2,2 & 2,7 & 0,2 & 0,5 \\
\hline Crepis biennis $\mathrm{L}$. & 0,2 & 0,1 & 0,0 & 0,2 \\
\hline Crepis vesicaria subsp. taraxacifolia (Thuill.) Thell. & 0,8 & 0,3 & 0,0 & 0,6 \\
\hline Dactylis glomerata L. & 35,6 & 6,4 & 0,8 & 4,3 \\
\hline Daucus carota $\mathrm{L}$. & 1,7 & 1,3 & 6,0 & 0,4 \\
\hline Echium vulgare $\mathrm{L}$. & 0,0 & 0,0 & 0,2 & 0,0 \\
\hline Elymus repens (L.) Gould & 19,9 & 0,0 & 0,0 & 0,0 \\
\hline Erigeron аппииs (L.) Desf. & 0,0 & 0,0 & 0,7 & 0,3 \\
\hline Erodium cicutarium (L.) L’Hér. & 0,2 & 0,0 & 0,0 & 0,0 \\
\hline Festuca arundinacea Schreb. & 20,0 & 20,2 & 6,0 & 6,5 \\
\hline Festuca ovina $\mathrm{L}$. & 0,0 & 0,0 & 0,0 & 6,7 \\
\hline Festuca pratensis Huds. s.str. & 3,4 & 0,0 & 10,2 & 0,4 \\
\hline Festuca rubra aggr. & 0,0 & 0,0 & 19,5 & 6,7 \\
\hline Galium mollugo aggr. & 5,7 & 7,5 & 0,0 & 6,9 \\
\hline
\end{tabular}


Tableau 2 (suite 1). Recouvrement moyen des espèces végétales inventoriées dans les quadrats $x 2$ dates $x$ 3SPB par catégorie de SPB (voir Tableau 1) - Average overlay of plant species inventoried in quadrats $x 2$ dates $x$ 3SPB by category of BPS (see Table 1).

\begin{tabular}{|c|c|c|c|c|}
\hline \multirow{2}{*}{$\begin{array}{l}\text { Espèce végétale } \\
\text { (www.infoflora.ch/fr) }\end{array}$} & \multicolumn{4}{|c|}{ Recouvrement moyen $(\%)$} \\
\hline & PQI & PQII & GQI & GQII \\
\hline Galium verum L. s.str. & 0,2 & 5,6 & 1,0 & 0,0 \\
\hline Geranium dissectum $\mathrm{L}$. & 1,7 & 0,7 & 0,0 & 0,0 \\
\hline Geranium molle L. & 1,7 & 0,4 & 0,0 & 0,0 \\
\hline Geranium pusillum L. & 0,0 & 1,3 & 0,0 & 0,0 \\
\hline Glechoma hederacea L. s.str. & 0,2 & 0,0 & 0,0 & 0,0 \\
\hline Helictotrichon pubescens (Huds.) Pilg. & 0,0 & 1,6 & 1,8 & 6,9 \\
\hline Heracleum sphondylium L. s.str. & 6,7 & 0,0 & 0,0 & 0,0 \\
\hline Holcus lanatus L. & 7,8 & 16,4 & 17,3 & 8,5 \\
\hline Hypericum perforatum L. s.str. & 0,0 & 0,0 & 0,0 & 0,3 \\
\hline Hypochaeris radicata $\mathrm{L}$. & 0,0 & 0,0 & 0,5 & 1,9 \\
\hline Knautia arvensis (L.) Coult. & 0,3 & 1,8 & 0,0 & 2,1 \\
\hline Lathyrus pratensis $\mathrm{L}$. & 0,0 & 1,2 & 0,0 & 0,0 \\
\hline Leontodon hispidus L. & 0,7 & 0,0 & 0,2 & 4,3 \\
\hline Leucanthemum vulgare Lam. & 0,0 & 0,3 & 5,1 & 0,0 \\
\hline Lolium multiflorum Lam. & 1,3 & 0,0 & 0,0 & 0,0 \\
\hline Lolium perenne L. & 1,8 & 2,4 & 0,3 & 0,3 \\
\hline Lotus corniculatus L. & 3,7 & 4,4 & 5,3 & 3,1 \\
\hline Medicago lupulina $\mathrm{L}$. & 0,5 & 0,9 & 0,0 & 0,8 \\
\hline Medicago sativa $\mathrm{L}$. & 3,1 & 0,0 & 0,3 & 0,0 \\
\hline Myosotis arvensis (L.) Hill & 0,2 & 1,1 & 0,4 & 0,0 \\
\hline Onobrychis viciifolia Scop. & 0,0 & 11,7 & 0,0 & 14,0 \\
\hline Origanum vulgare L. & 0,2 & 0,0 & 0,0 & 0,0 \\
\hline Papaver rhoeas L. & 0,0 & 0,0 & 0,0 & 0,2 \\
\hline Petrorhagia prolifera (L.) P.W.Ball \& Heywood & 0,0 & 0,0 & 0,0 & 0,1 \\
\hline Phleum pratense aggr. & 0,3 & 0,0 & 0,0 & 0,0 \\
\hline Plantago lanceolata $\mathrm{L}$. & 3,6 & 14,1 & 6,2 & 7,4 \\
\hline Plantago media $\mathrm{L}$. & 0,3 & 0,0 & 0,0 & 0,0 \\
\hline Pоа аппиа $\mathrm{L}$. & 0,0 & 0,0 & 5,8 & 0,0 \\
\hline Poa pratensis $\mathrm{L}$. & 4,0 & 4,7 & 4,0 & 1,7 \\
\hline Poa trivialis L. s.str. & 15,3 & 0,0 & 9,2 & 1,3 \\
\hline Potentilla reptans $\mathrm{L}$. & 7,5 & 0,2 & 8,3 & 0,0 \\
\hline Prunella vulgaris L. & 0,0 & 0,3 & 4,7 & 0,4 \\
\hline Prunus avium (L.) L. & 0,0 & 0,1 & 0,0 & 0,0 \\
\hline Ranunculus acris subsp. friesianus (Jord.) Rouy \& Foucaud & 10,0 & 1,9 & 13,6 & 0,5 \\
\hline Ranunculus bulbosus L. & 1,6 & 5,0 & 0,0 & 0,9 \\
\hline Rhinanthus alectorolophus (Scop.) Pollich & 5,8 & 4,0 & 4,8 & 0,3 \\
\hline Rumex acetosa $\mathrm{L}$. & 0,8 & 1,6 & 0,5 & 0,9 \\
\hline Rumex crispus $\mathrm{L}$. & 0,0 & 0,0 & 0,8 & 0,0 \\
\hline
\end{tabular}


Tableau 2 (suite 2). Recouvrement moyen des espèces végétales inventoriées dans les quadrats $x 2$ dates $\times 3$ SPB par catégorie de SPB (voir Tableau 1) - Average overlay of plant species inventoried in quadrats $\times 2$ dates $\times 3$ SPB by category of BPS (see Table 1).

\begin{tabular}{|c|c|c|c|c|}
\hline \multirow{2}{*}{$\begin{array}{l}\text { Espèce végétale } \\
\text { (www.infoflora.ch/fr) }\end{array}$} & \multicolumn{4}{|c|}{ Recouvrement moyen $(\%)$} \\
\hline & $\overline{\mathrm{PQI}}$ & PQII & GQI & GQII \\
\hline Rumex obtusifolius L. & 0,0 & 0,0 & 0,2 & 0,0 \\
\hline Salvia pratensis $\mathrm{L}$. & 4,8 & 0,0 & 0,0 & 8,1 \\
\hline Sanguisorba minor Scop. s.str. & 0,0 & 17,1 & 0,0 & 17,5 \\
\hline Scabiosa columbaria L. s.str. & 0,0 & 0,0 & 0,0 & 3,7 \\
\hline Silene pratensis Godr. & 0,0 & 0,0 & 0,2 & 0,1 \\
\hline Silene vulgaris (Moench) Garcke s.str. & 0,0 & 0,2 & 0,0 & 0,3 \\
\hline Taraxacum officinale aggr. & 1,7 & 0,2 & 7,0 & 1,0 \\
\hline Tragopogon pratensis subsp. orientalis (L.) Celak. & 0,3 & 0,8 & 0,5 & 0,5 \\
\hline Trifolium campestre Schreb. & 1,0 & 0,6 & 0,0 & 0,5 \\
\hline Trifolium incarnatum L. s.str. & 0,0 & 0,0 & 0,0 & 0,2 \\
\hline Trifolium pratense L. s.str. & 3,1 & 4,3 & 6,5 & 2,9 \\
\hline Trifolium repens $\mathrm{L}$. & 1,2 & 0,5 & 5,9 & 0,5 \\
\hline Trisetum flavescens (L.) P.Beauv. & 14,3 & 4,9 & 5,5 & 7,8 \\
\hline Valerianella locusta (L.) Laterr. & 0,0 & 0,5 & 0,0 & 0,0 \\
\hline Veronica arvensis L. & 1,5 & 0,9 & 0,4 & 0,1 \\
\hline Veronica chamaedrys L. & 0,0 & 0,8 & 0,0 & 0,0 \\
\hline Veronica persica Poir. & 0,0 & 0,1 & 0,0 & 0,0 \\
\hline Vicia hirsuta (L.) Gray & 0,0 & 0,0 & 0,2 & 0,2 \\
\hline Vicia sativa subsp. nigra (L.) Ehrh. & 0,8 & 2,6 & 1,2 & 0,8 \\
\hline Viola arvensis Murray & 0,0 & 0,0 & 0,0 & 0,1 \\
\hline
\end{tabular}

Tableau 3. Recouvrement moyen des espèces végétales en fleurs dans les quadrats $x 2$ dates $\times$ 3SPB par catégorie de SPB (voir Tableau 1) - Average overlay of flowering plant inventoried in quadrats $x 2$ dates $x 3 S P B$ by category of BPS (see Table 1).

\begin{tabular}{lllll}
\hline Catégorie & PQI & PQII & GQI & GQII \\
\hline Recouvrement moyen en fleurs (\%) & 8,42 & 21,54 & 19,33 & 14,71
\end{tabular}

\section{4. Échantillonnage des syrphes}

Deux méthodes complémentaires ont été utilisées :

- les pièges d'interception Malaise $(\mathrm{L}: 170 \mathrm{~cm}$, $\mathrm{h}: 170 \mathrm{~cm}, \quad 1: 110 \mathrm{~cm}$ ) (Malaise, 1937), qui échantillonnent les syrphes adultes qui fréquentent les SPB en volant ;

- les pièges à émergence, qui échantillonnent les syrphes adultes émergeant du sol ou de la strate herbacée et ayant donc effectué leur développement larvaire dans les SPB.

Il s'agit de pièges Malaise clos, couvrant $1,8 \mathrm{~m}^{2}$ au sol. Les pièges proviennent de $B \& S$ Entomological
Services, Co. Armagh, Irlande du nord, GB. Les premiers échantillonnent un assemblage d'espèces plus abondant et diversifié pouvant provenir d'habitats situés hors des SPB. Ils renseignent sur le rôle de la SPB comme zone de nourrissage potentielle pour l'entomofaune du paysage avoisinant. Les seconds fournissent une mesure des espèces réellement hébergées par les SPB. Sur chacun des sites, deux pièges à émergence et un piège Malaise ont été installés. Le piège Malaise a été placé vers le centre de la SPB de façon à gêner le moins possible le travail des exploitants (fauche) et les deux pièges à émergence à environ $2 \mathrm{~m}$ de part et d'autre du piège Malaise afin de ne pas entraver son emprise (Figure 2). Les pièges 

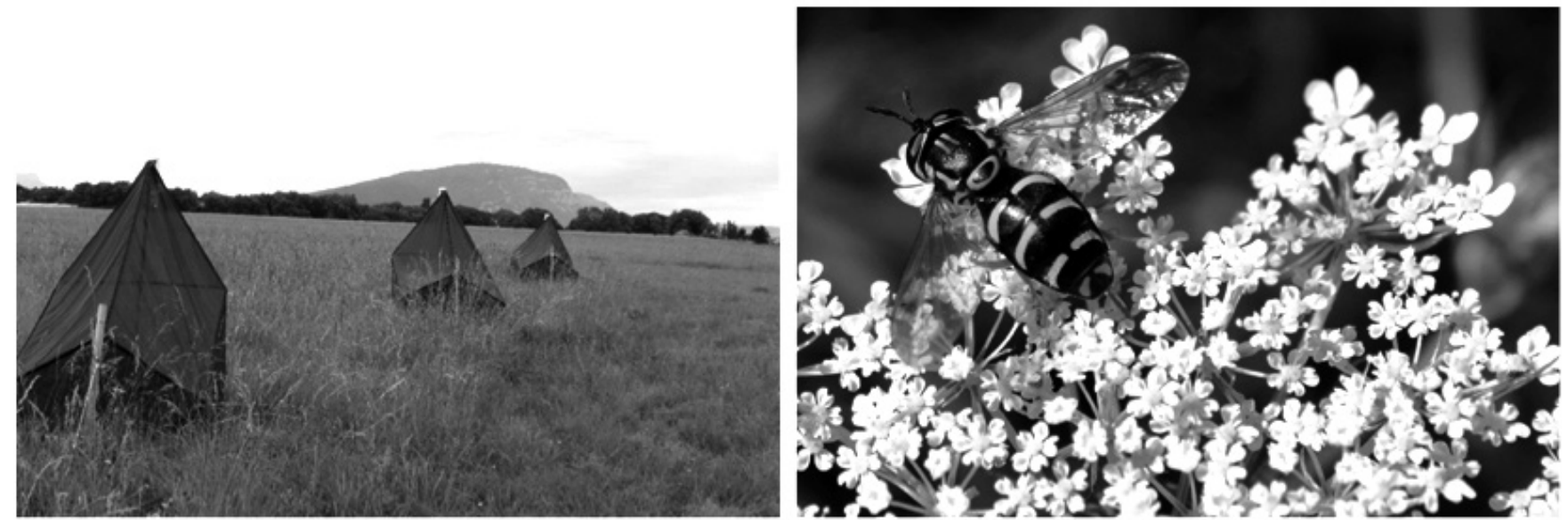

Figure 2. Pièges : tente Malaise au centre et pièges à émergence de part et d'autre (à gauche), Chrysotoxum faestivum sur Daucus carota (à droite) - Malaise and emergency traps (left) Chrysotoxum faestivum on Daucus carota (right).

Photos : Marie Bessat.

Malaise ont fonctionné de début avril à fin juin 2017 et les pièges à émergence de début avril à fin aout 2017. Les flacons de capture, remplis d'éthanol à $75 \%$, ont été vidés toutes les deux semaines (six relevés pour les pièges Malaise et neuf pour les émergences). Le tri et la détermination (selon Van Veen, 2004 et Speight \& Castella, 2015) ont été effectués en laboratoire sous loupe binoculaire.

\subsection{Utilisation de la méthode Syrph-the-Net}

La base de données StN (Speight, 2015) contient une matrice associant 926 espèces de syrphes européens à 325 catégories d'habitats définis sur la base du système européen CORINE faisant essentiellement appel à des groupements phyto-sociologiques (Devillers et al., 1991). À l'intérieur d'un espace géographique donné pour lequel une liste d'espèces connue existe, dans notre cas la liste du canton de Genève de Speight et al. (2019), la base de données peut être utilisée afin de produire une liste d'espèces potentielles pour un ou plusieurs habitats identifiés selon la liste des habitats $\mathrm{StN}$, ce sont les espèces «prédites ». Elles peuvent être utilisées afin de mesurer un niveau d'intégrité écologique (IE) d'un habitat ou d'un site (ensemble d'habitats). L'IE est dans ce cas définie comme le nombre d'espèces «au rendez-vous », c'est-à-dire prédites et effectivement observées, exprimé en pourcentage du nombre d'espèces prédites. Speight \& Castella (2001), Burgio \& Sommaggio (2007), Sarthou \& Speight (2013) ont proposé et utilisé des seuils d'interprétation de ces valeurs : $<20 \%$ - IE très faible, ]20 - 40\%] - IE faible, ]40 - 50\%] - IE moyenne, ]50 - $75 \%$ ] - IE bonne, ]75 - 85\%] - IE très bonne, $>85 \%$ - IE excellente. Ce principe a été utilisé ici de deux façons. Compte tenu de la variabilité botanique inter-SPB, il a été utilisé pour comparer la liste d'espèces obtenues en émergence avec les listes d'espèces genevoises prédites pour trois catégories d'habitats StN considérées comme les plus proches des SPB étudiées : les prairies améliorées à foin ( $\mathrm{StN}$ 23213), les prairies non améliorées humides eutrophes/ mésotrophes (StN 231131) et les prairies sèches non améliorées calcaires (StN 231122). D'autre part, les espèces échantillonnées par les pièges Malaise ont été mises en relation avec les habitats inventoriés selon la typologie StN dans un rayon de $200 \mathrm{~m}$ autour des pièges.

\subsection{Analyses statistiques}

Les analyses statistiques et graphiques ont été réalisées à l'aide du logiciel R (R Development Core Team, 2008) et des jeux de fonctions ade4 (Chessel et al., 2004) et vegan (Oksanen et al., 2017).

Les comparaisons de richesses spécifiques et d'abondances entre les catégories du plan d'échantillonnage ont été réalisées à l'aide de tests de Wilcoxon non paramétriques pour des raisons d'homogénéité, compte tenu de la non-normalité de plusieurs séries de valeurs. Une Analyse en Composantes Principales (ACP) centrée a été réalisée afin de rechercher les catégories de traits des syrphes participant le plus à la discrimination des sites.

La quantité de plantes en fleurs en juin dans la FV présente sous les pièges à émergence a été mise en relation avec les richesses et abondances des syrphes émergents entre mi-juin et début aout à l'aide de tests de corrélation. La relation entre les traits de la syrphifaune 
émergente (mode trophique larvaire [herbivores, carnivores], voltinisme [univoltines, biovoltines, polyvoltines] et période de vol [février-mai, juin-aout septembre-novembre]) et le recouvrement des espèces végétales a été explorée à l'aide d'une analyse de co-inertie (testée avec un test de Monte-Carlo, à un seuil de $5 \%$ ) et de tests de corrélation.

$\mathrm{La}$ relation entre la syrphifaune volante et les caractéristiques des espèces végétales a été explorée à l'aide d'une analyse de co-inertie. Les traits des espèces végétales, extraits de la base de données BiolFlor (Kühn et al., 2004), sont un compromis entre ceux mentionnés le plus souvent dans la littérature comme influençant les syrphes et ceux disponibles dans cette base de données : les familles (Fabaceae, Asteraceae), la durée de floraison en mois $(<4,4-5, \geq 6)$, le type biologique (hémicryptophyte, géophyte, thérophyte), la récompense florale (nectar, pollen), la couleur des fleurs (jaune, blanc, bleu, violet-rose). Sur la base de l'analyse de co-inertie, des corrélations entres les traits floraux et les abondances et richesses syrphidologiques ont ensuite été calculées. L'IE a également été mise en relation avec certains traits floristiques via des tests de corrélation. Les seuils de significativité ont été considérés à $5 \%$, y compris ceux des tests de MonteCarlo, associés aux analyses de co-inertie.

\section{RÉSULTATS}

\subsection{Quelle syrphifaune se développe au stade larvaire dans les SPB ?}

Deux-cent-treize individus appartenant à 20 espèces de syrphes ont été capturés par la totalité des pièges à émergence sur l'ensemble de la période d'échantillonnage (Tableau 4). La taille des SPB n'affecte significativement ni la richesse spécifique (test de Wilcoxon, $\mathrm{w}: 18,5 ; p: 1$ ), ni l'abondance (test de Wilcoxon, w : $24 ; p: 0,37)$ des syrphes. De même, la qualité botanique n'a d'effet significatif ni sur la richesse spécifique (test de Wilcoxon, $\mathrm{w}: 16,5 ; p$ : 0,87 ) ni sur l'abondance (test de Wilcoxon, w : 17,5; $p: 1)$.

Les espèces de syrphes ayant émergé des SPB sont majoritairement bi- et polyvoltines $(70 \%)$, ont des larves carnivores $(95 \%)$ et vivant à la surface des plantes herbacées $(70 \%)$. L'ACP ordonne les 12 SPB essentiellement en fonction des proportions d'individus appartenant à ces quatre catégories de traits les plus représentées, qui augmentent de droite à gauche de l'axe F1 (Figure 3).

Parmi les trois catégories StN de prairies comparées, ce sont les espèces associées à la catégorie

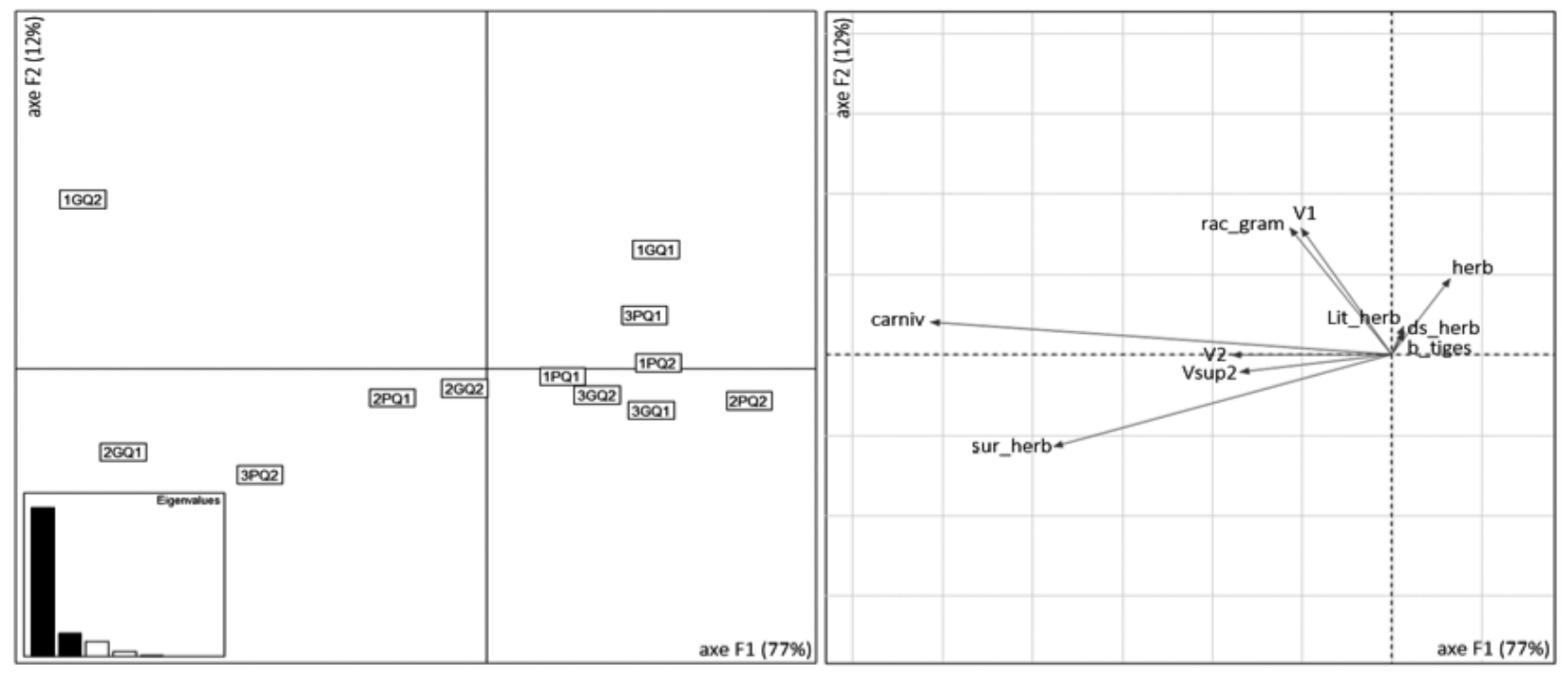

Figure 3. ACP centrée des 12 sites positionnés (voir Tableau 1) en fonction des traits fonctionnels larvaires caractérisant la syrphifaune émergente - Centered PCA of the 12 sites according to the larval functional traits characterizing the emergent syrphids (see Table 1).

Les 10 modalités de traits considérées sont les suivantes : mode trophique larvaire: carnivores (carniv) et herbivores (herb), zone de développement larvaire : base des tiges (b_tiges), racines des graminées (rac_gram), sur les herbacées (sur_herb), dans les herbacées (ds_herb) et dans la litière herbacée (Lit_herb) et voltinisme: univoltines (V1), bivoltines (V2), polyvoltines (Vsup2) - The 10 traits are as follows: food type larvae: living animals (carniv) and living plants (herb), larval activity zone: terrestrial: base of the stems (b_tiges), roots of the grass (rac_gram): on herb-layer plants (sur_herb), in herb-layer plants (ds_herb) and litter (Lit_herb), and the number of generations per year: univoltines (V1), bivoltines (V2), polyvoltines (Vsup2). 


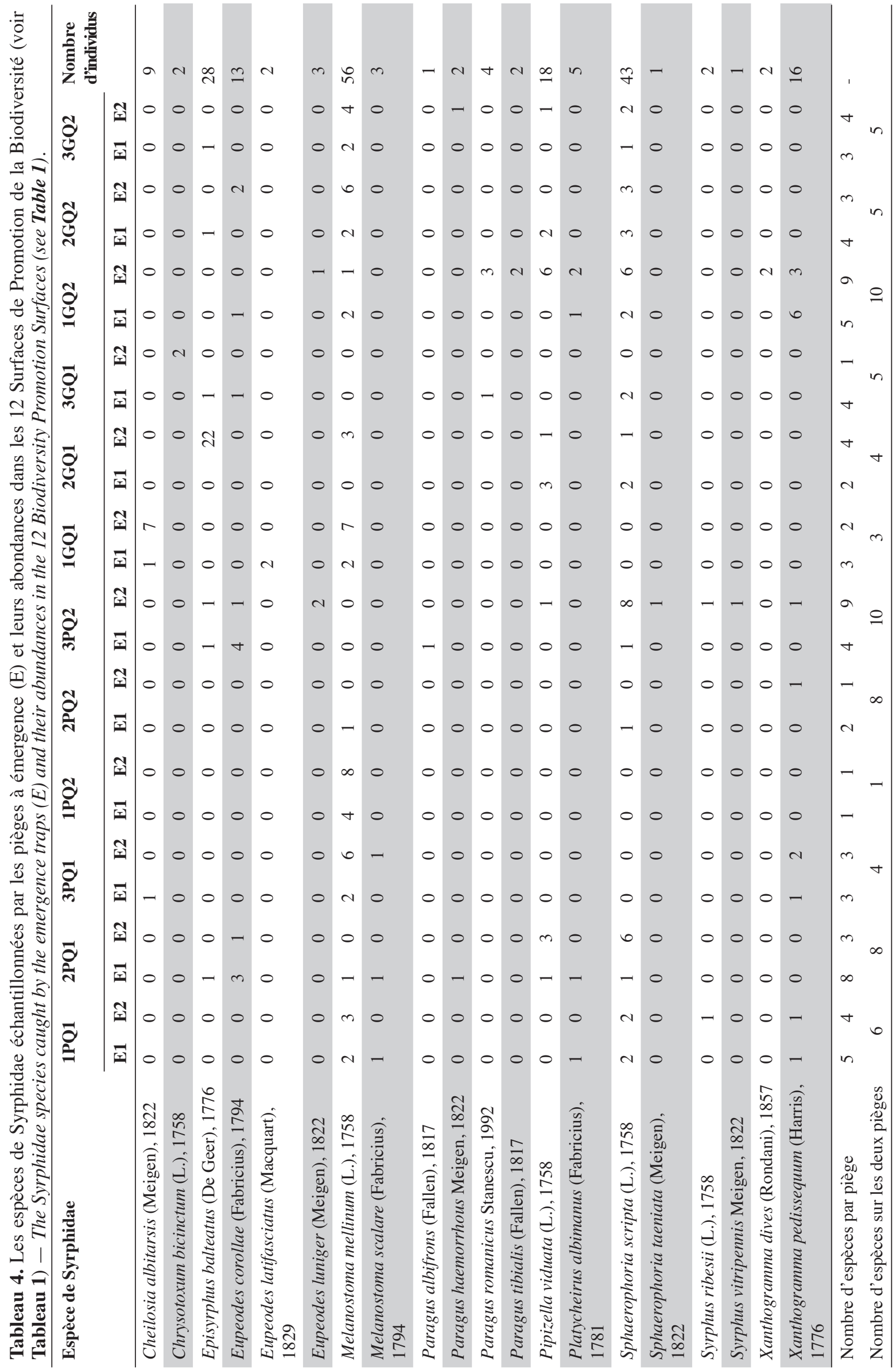


« prairies améliorées à foin » qui montrent la meilleure adéquation avec la liste d'espèces observées sur les sites. Le cumul des pièges à émergence a conduit à l'échantillonnage de près de $70 \%$ des espèces attendues pour cet habitat à Genève (neuf des treize espèces prédites ont été échantillonnées : Chrysotoxum bicinctum, Episyrphus balteatus, Eupeodes corollae, Melanostoma mellinum, Platycheirus albimanus, Sphaerophoria scripta, Cheilosia albitarsis, Melanostoma scalare et Syrphus ribesii). Les six premières espèces de cette liste sont aphidiphages au stade larvaire. Les caractéristiques larvaires de ces espèces «au rendez-vous » montrent une nette dominance de certains microhabitats : les espèces se développant sur les grandes herbacées, sur les herbes basses, dans la litière herbacée du sol et dans la couche superficielle du sol sont mieux représentées que celles se développant à l'intérieur des tissus végétaux. Par opposition, les quatre espèces « manquantes » (prédites mais non observées): Cheilosia pagana, Cheilosia vernalis, Platycheirus clypeatus et Sphaerophoria interrupta sont essentiellement univoltines, leurs larves sont herbivores et se développent à l'intérieur des tissus des végétaux.

\subsection{Composition végétale des SPB et syrphifaune émergente}

La richesse spécifique et l'abondance des syrphes émergents de juin à aout sont corrélées positivement à l'abondance moyenne des plantes en fleurs dans les SPB au mois de juin (Figure 4). L'analyse de

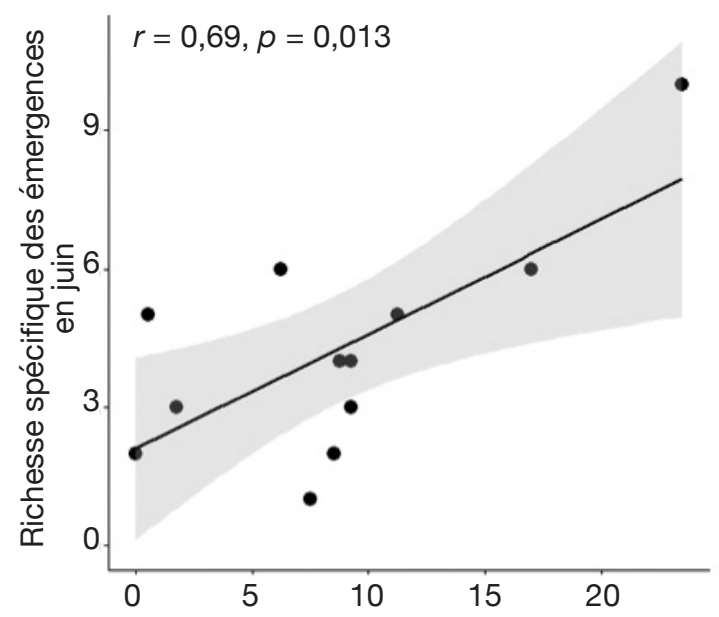

Abondance moyenne des plantes en fleurs en juin co-inertie entre les recouvrements des espèces végétales et les caractéristiques fonctionnelles des syrphes émergents révèle une relation significative entre ces deux ensembles de données (test de MonteCarlo, $p: 0,024)$. Une analyse par variable montre que seuls les recouvrements d'Achillea millefolium et de Tragopogon pratensis sont significativement corrélés au pourcentage d'individus carnivores émergents des SPB (Figure 5).

\subsection{Quelle syrphifaune fréquente les SPB au stade adulte?}

Quatre-mille-sept-cent-quatre individus de syrphes appartenant à 66 espèces ont été capturés dans l'ensemble des 12 pièges Malaise, entre début avril et fin juin 2017 (Tableau 5). La taille des SPB n'affecte significativement ni la richesse spécifique (test de Wilcoxon, w: $420 ; p: 0,66$ ), ni l'abondance (test de Wilcoxon, w: $18 ; p: 1)$ des syrphes. De même, la qualité botanique n'a d'effet significatif ni sur la richesse spécifique (test de Wilcoxon, w: 436; $p$ : 0,84 ), ni sur l'abondance (test de Wilcoxon, w : 15,5; $p: 0,75)$.

Deux espèces dominaient largement l'échantillonnage : S. scripta (53\% des individus) et M. mellinum (18\% des individus). D'autres espèces, moins abondantes, mais assez fréquentes étaient présentes dans les échantillons: E.balteatus, Sphaerophoria taeniata, Pipizella viduata, E. corollae, S. ribesii et Paragus haemorrhous. Près de la moitié des espèces capturées dans les pièges Malaise ont des larves prédatrices.

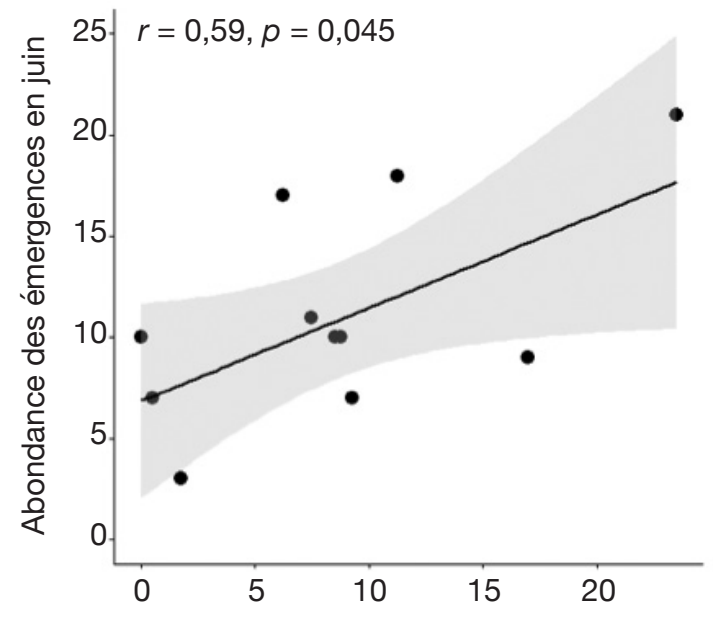

Abondance moyenne des plantes en fleurs en juin

Figure 4. Corrélations entre l'abondance moyenne des plantes en fleurs en juin et la richesse spécifique des Syrphidae dans les pièges à émergence après la fauche dans chaque site (à gauche) et entre l'abondance moyenne des plantes en fleurs en juin et le nombre d'individus piégés dans les pièges à émergence de chacun des sites après la fauche (à droite) - Correlations between the mean abundance of plants in flower in June and the syrphid species richness in the emergence traps measured after the grass cut (left) and between the mean abundance of plants in flower in June and the number of syrphid individuals in the emergence traps after the grass cut (right). 


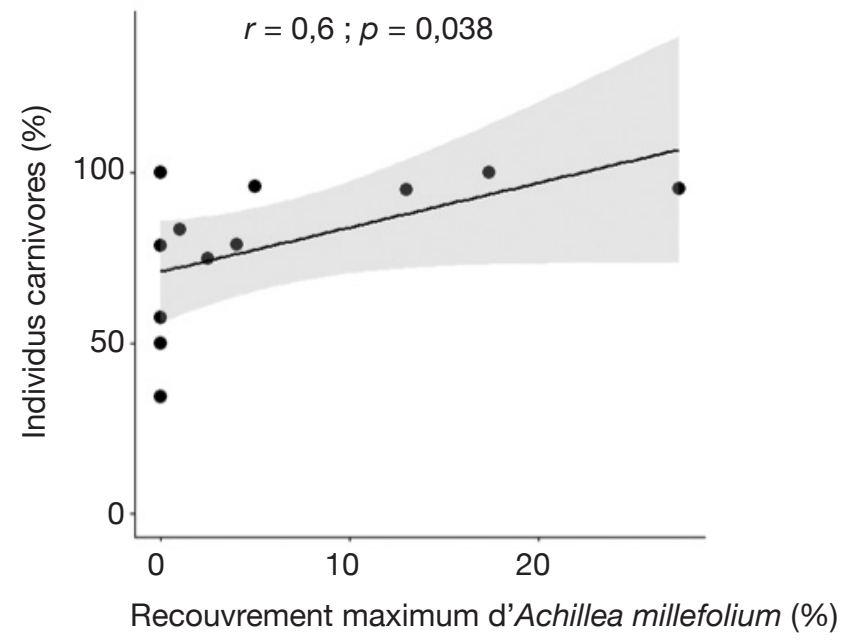

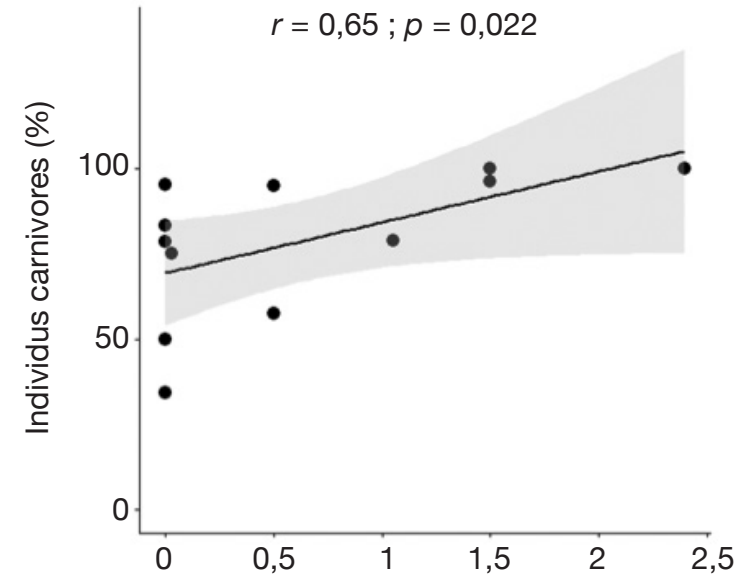

Recouvrement maximum de Tragopogon pratensis (\%)

Figure 5. Corrélations entre le recouvrement d'Achillea millefolium et le pourcentage d'individus à larves carnivores (à gauche) et le recouvrement de Tragopogon pratensis et le pourcentage d'individus à larves carnivores (à droite) - Correlations between Achillea millefolium cover and the percentage of individuals with carnivorous larvae (left) and between Tragopogon pratensis cover and the percentage of individuals with carnivorous larvae (right).

L'analyse des habitats auxquels sont associées les espèces échantillonnées dans les pièges Malaise montre que $20 \%$ d'entre elles sont potentiellement associées aux SPB elles-mêmes, les $80 \%$ restants étant associés à des habitats périphériques, principalement des milieux cultivés pouvant être identifiés dans un rayon de $200 \mathrm{~m}$ à $1 \mathrm{~km}$ autour des pièges. Dans les douze SPB considérées collectivement, $92 \%$ des espèces associées aux SPB (12 espèces, sur les 13 prédites pour les SPB) ont été échantillonnées, ce qui témoigne d'une excellente IE pour l'habitat



Recouvrement des fleurs jaunes (\%)
« prairies améliorées à foin » à l'échelle du canton de Genève. Cette IE est positivement et significativement corrélée à deux traits floristiques: le recouvrement des espèces végétales possédant des fleurs jaunes $(r$ : $0,71, p: 0,001)$, ainsi que le recouvrement des espèces végétales dont les fleurs produisent du nectar $(r$ : $0,75 ; p: 0,005)$ (Figure 6). Par ailleurs, une analyse par variable montre que seules deux modalités de traits sont en particulier corrélées significativement et positivement aux données d'abondance et de richesse spécifique : l'augmentation du recouvrement des fleurs

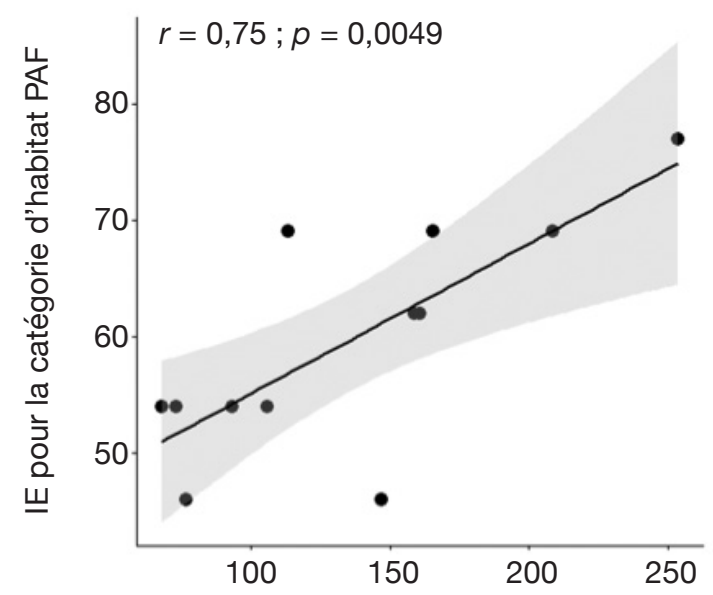

Recouvrement des fleurs produisant du nectar (\%)

Figure 6. Corrélations entre le recouvrement des espèces végétales possédant des fleurs jaunes et l'intégrité écologique (IE) pour la catégorie d'habitat «prairies améliorées à foin »(PAF) de chacun des sites (à gauche) et entre le recouvrement des espèces végétales dont les fleurs produisent du nectar et l'intégrité écologique pour la même catégorie d'habitat de chacun des sites (à droite) - Correlations between the cover of plants with yellow flowers and the ecological integrity (IE) of improved grassland (PAF) (left) and between the cover of nectar-producing plants and the ecological integrity of improved grassland (right). 


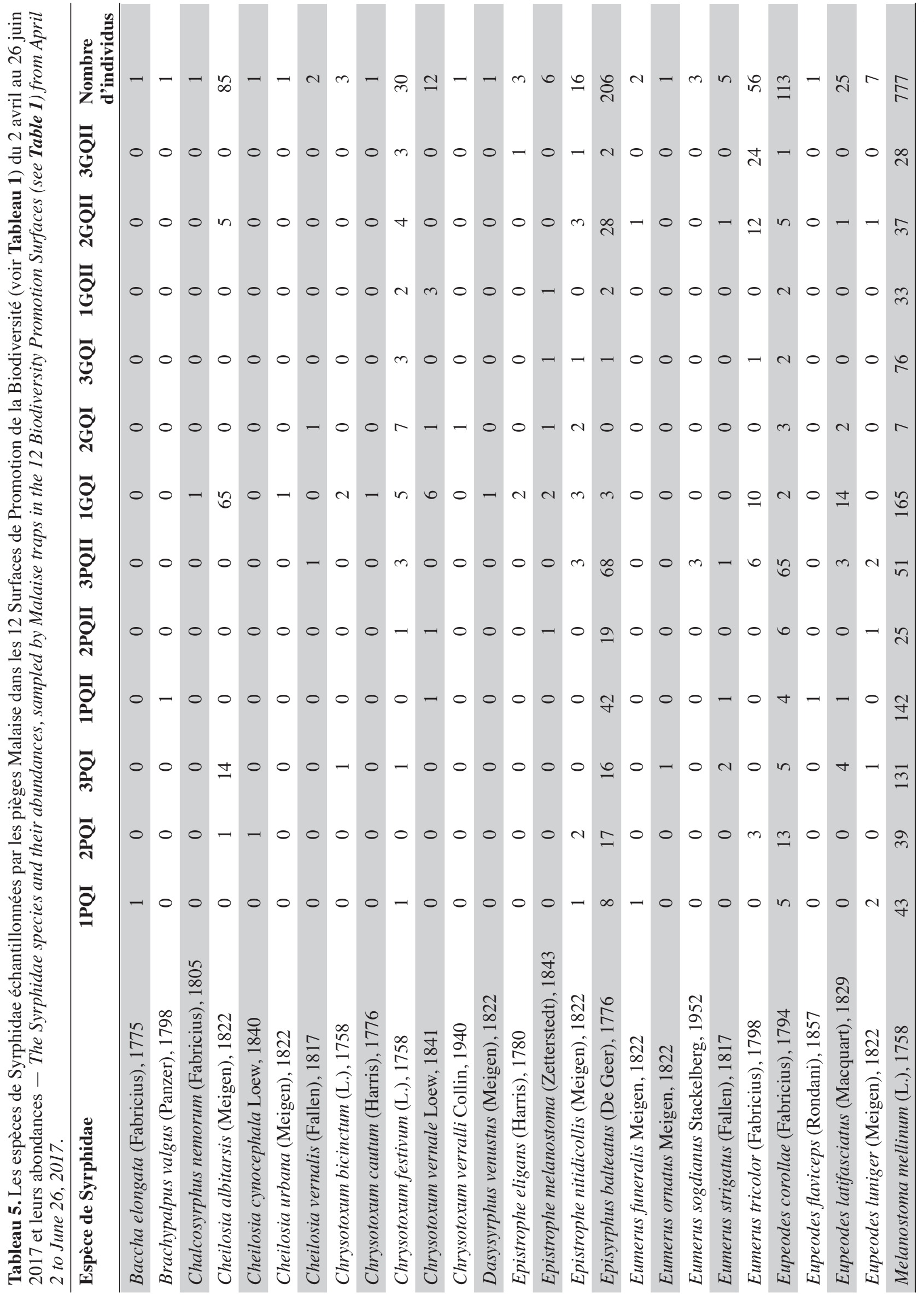




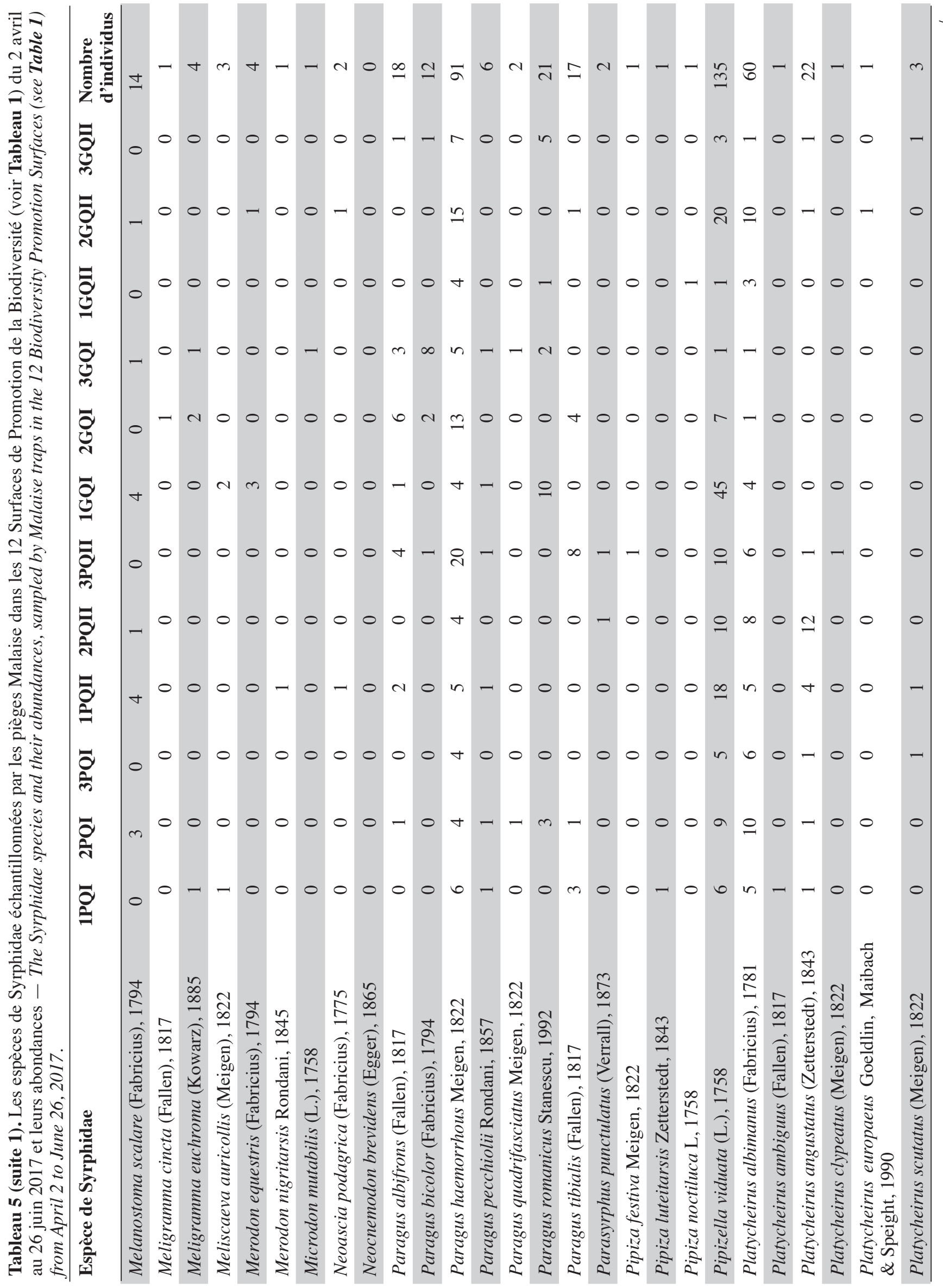




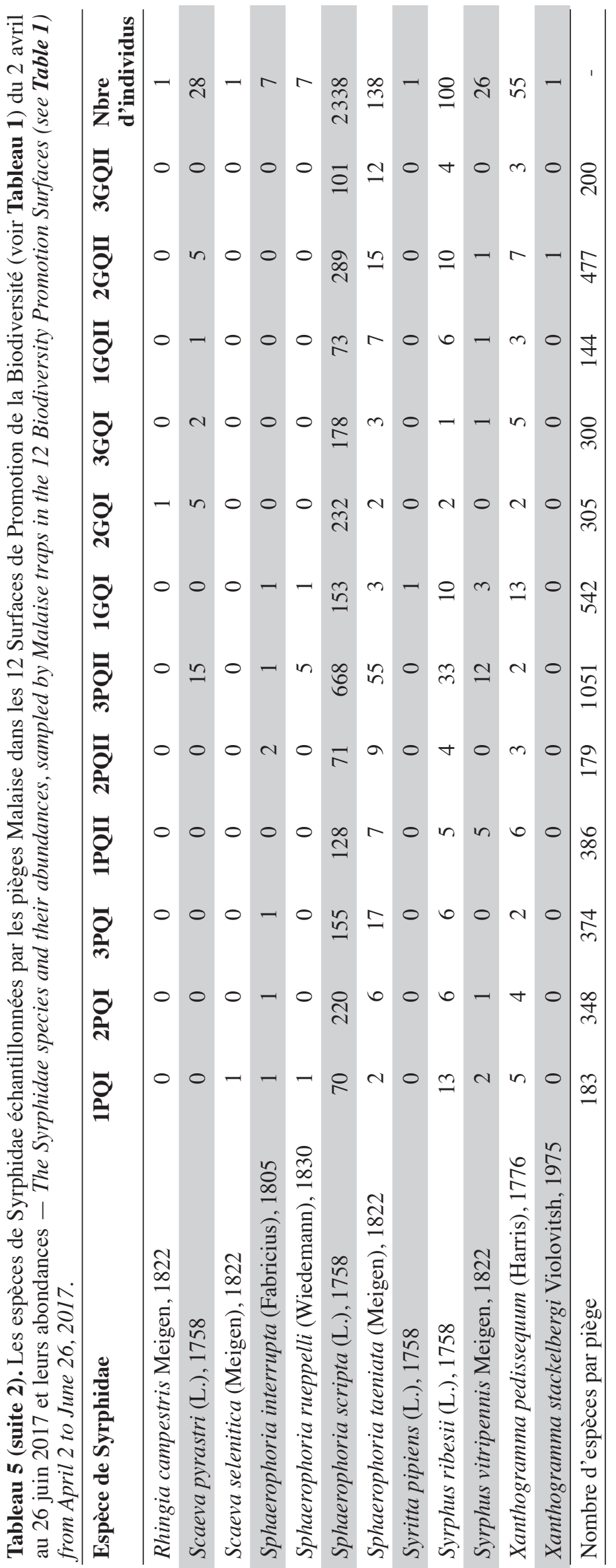

de couleur rose-violette est corrélée positivement à l'augmentation du nombre total d'individus capturés dans les pièges Malaise $(r: 0,7 ; p$ : $0,012)$. Le recouvrement des plantes produisant du nectar est corrélé positivement, mais marginalement $(r: 0,56 ; p: 0,058)$ à la richesse spécifique en syrphes (Figure 7).

\subsection{Syrphifaunes émergente et «passante »}

La confrontation des assemblages de syrphes échantillonnés par les deux méthodes peut être effectuée en exprimant les résultats par SPB $(n=12)$. Elle montre des résultats convergents: les richesses en Syrphidae volants ou émergents, exprimées en nombre total d'espèces échantillonnées ou en nombre d'espèces effectivement associées à la catégorie d'habitat StN «prairies améliorées à foin » ne sont pas significativement corrélées aux richesses végétales (tous les coefficients de corrélation avec $p>0,05)$. Par ailleurs, les richesses en syrphes volants et émergents ne sont pas corrélées, exprimées en valeurs brutes $(r=0,17 ; p=0,6)$ ou en pourcentage de représentation des espèces potentiellement associées à la catégorie d'habitat $\mathrm{StN}$ «prairies améliorées à foin » $(r=0,04 ; p=$ $0,9)$. Toutefois, 19 des 20 espèces récoltées en émergence ont été récoltées également dans les pièges Malaise où elles ne représentent que $29 \%$ des espèces, mais $94 \%$ des individus. Pour ces 20 espèces, la corrélation entre leurs abondances relatives dans les deux types de pièges est significative $(r=0,61 ; p=0,005)$. Les trois espèces qui dominent les captures en émergence (M. mellinum $26 \%$, S. scripta $20 \%$, E. balteatus $13 \%$ ) sont les mêmes que dans les pièges Malaise (respectivement $17 \%, 53 \%$ et $4 \%$ des captures).

\section{DISCUSSION}

\subsection{Qualité et taille des SPB}

Nos résultats montrent que l'échantillonnage de 12 SPB avec un seul piège Malaise par SPB (et non pas deux, comme recommandé par Speight et al. [2000]), dans un paysage à dominance agricole, permet d'échantillonner plus d'un quart de la faune syrphidologique genevoise sur une période de trois mois, ainsi que $92 \%$ des espèces pouvant être attendues localement en association avec l'habitat prairial échantillonné. Cependant, ni la qualité botanique, ni la taille des SPB n'ont de relation avec les métriques utilisées pour décrire les communautés de syrphes se développant au 

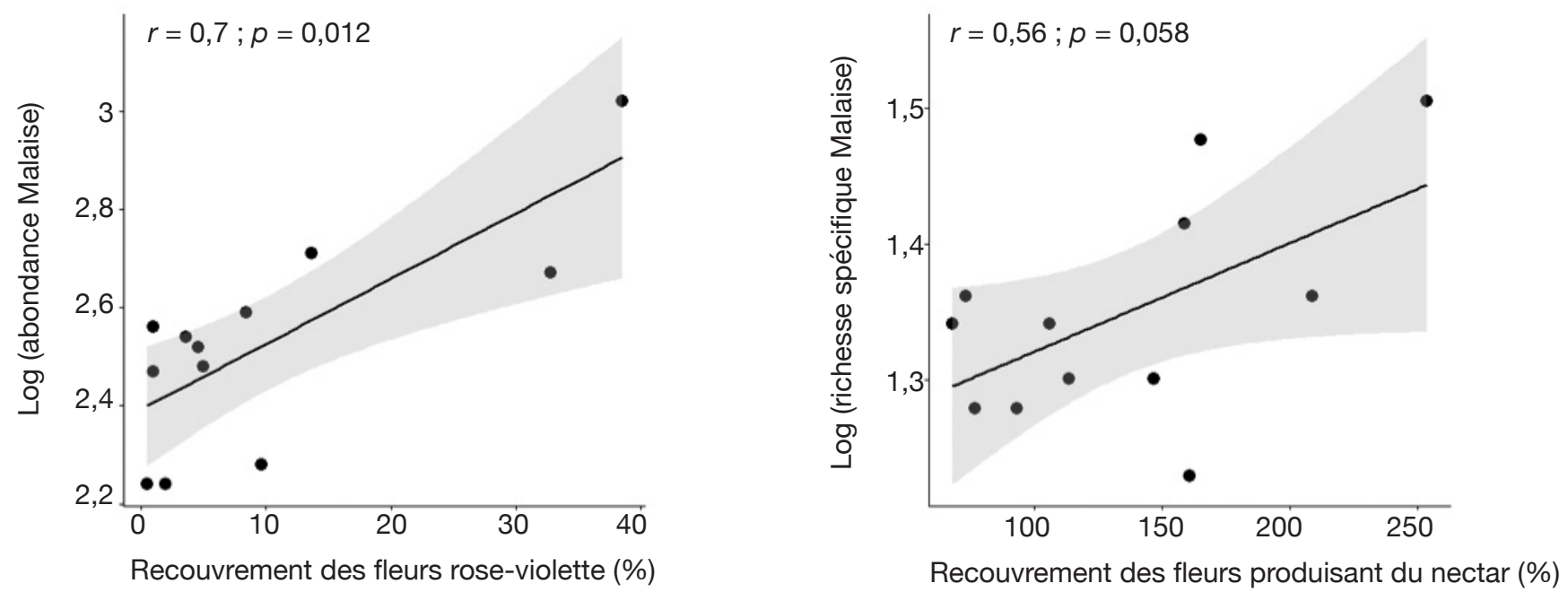

Figure 7. Corrélations entre le recouvrement des espèces végétales possédant des fleurs rose-violette et les abondances log-transformées des syrphes dans les pièges Malaise (à gauche) et entre le recouvrement des espèces végétales dont les fleurs produisent du nectar et les richesses spécifiques log-transformées des syrphes échantillonnés par les pièges Malaise (à droite) - Correlations between the cover of plants with pink-purple flowers and the log-transformed abundances of syrphids in the Malaise traps (left) and between the cover of plants with nectar-producing flowers and the log-transformed syrphid species richness in the Malaise traps (right).

stade larvaire dans les SPB ou les fréquentant au stade adulte. La distinction des deux niveaux de qualité des $\mathrm{SPB}$, basée sur des critères botaniques, ne se répercute pas sur la faune syrphidologique. Concernant ce dernier point, nous montrons toutefois des relations positives entre certaines métriques syrphidologiques (richesse et abondance des espèces émergentes, intégrité écologique de l'habitat prairial) et des métriques botaniques (abondance des plantes en fleurs, recouvrement des plantes à fleurs jaunes et des fleurs nectarifères), non directement prises en compte par la classe de qualité définie dans la norme suisse. En ce sens, nos résultats peuvent être rapprochés de ceux de Sutherland et al. (2001) et Blaauw \& Isaacs (2014) qui ne trouvent pas de relations entre la taille de surfaces fleuries et l'abondance des syrphes, mais une relation positive entre l'abondance des plantes en fleurs dans ces structures et celle des syrphes. Il convient cependant de noter que ces études diffèrent de la nôtre par la plus faible taille des surfaces considérées et des méthodes d'échantillonnage entomologique très différentes.

\subsection{Diversité fonctionnelle}

Nous montrons que les SPB étudiées sont des milieux prairiaux permettant le soutien et le développement d'une syrphifaune aphidiphage ayant donc un potentiel de lutte biologique. On peut ainsi supposer qu'elles peuvent, à l'échelle du paysage local, contribuer à augmenter le pool d'espèces bénéfiques, notamment par le biais d'effets de débordement (spillover) vers les cultures adjacentes, comme cela a été montré pour les bandes fleuries (Haenke, 2009 ; Hatt et al., 2017) et y diminuer ainsi les ravageurs.

Nous montrons que la quantité d'individus aphidiphages émergents est positivement corrélée à l'augmentation des recouvrements d'A. millefolium et de T. pratensis aggr. Plusieurs études ont montré le rôle positif que joue A.millefolium dans l'augmentation de la richesse et de l'abondance en syrphes, essentiellement pour les espèces aphidiphages (George et al., 2010), en lien avec la capacité de cette espèce à héberger un grand nombre de pucerons (Carrié et al., 2012). Certains stimuli floraux ainsi que la présence de pucerons sur certaines espèces végétales sont des facteurs permettant d'attirer les syrphes. Ces résultats suggèrent donc que l'implantation de certaines espèces végétales dans les SPB pourrait jouer un rôle clé dans le maintien de services écosystémiques en contexte agricole, tels que la lutte contre les ravageurs des cultures ou la pollinisation (Carrié et al., 2012).

La quantité de fleurs dans une prairie est une composante essentielle pour les insectes pollinisateurs (Kleijn \& Van Langevelde, 2006; Haenke, 2009 ; Meyer et al., 2009). Beaucoup d'auteurs associent d'ailleurs la qualité d'une prairie à son abondance en fleurs (Kleijn \& Van Langevelde, 2006; Zurbrügg $\&$ Frank, 2006). Nous montrons que la quantité de plantes en fleurs au mois de juin est positivement liée avec la richesse et l'abondance des syrphes émergents. L'amalgame des périodes d'échantillonnage dans l'analyse induit nécessairement la prise en compte simultanée des individus dont les œufs ont été pondus l'année précédente (2016) et de ceux dont les œufs ont 
été pondus au printemps 2017. Un suivi temporel plus fin de la phénologie des fleurs et des espèces de syrphes sur l'ensemble de la saison permettrait d'analyser plus en détail la synchronisation et les relations entre espèces des deux groupes.

La ponte des syrphes aphidiphages dans les SPB ne dépend donc sans doute pas que de la quantité de fleurs présentes dans les prairies, mais également de la présence des pucerons nécessaires au développement larvaire (Almohamad et al., 2009). Si l'abondance et la richesse spécifique des syrphes émergents dépendent de la quantité des fleurs présentes dans les SPB au mois de juin, c'est également à cette période (15 juin) qu'a été fixée la date de fauche des prairies extensives en plaine. Une des principale « mesure réseau » utilisée et appliquée à l'ensemble des 12 SPB échantillonnées est le maintien de $10 \%$ de la surface non fauchée. Cette mesure semble donc fondamentale au vu des résultats obtenus, d'autant plus que les syrphes émergents dépendent ensuite des ressources florales au stade adulte. Ces résultats confirment que des SPB plus riches en fleurs constituent des milieux permettant de maximiser la visite par les syrphes adultes (Kleijn \& Van Langevelde, 2006 ; Haenke, 2009) et de favoriser le développement larvaire des espèces liées à ce type de milieux. En effet, les espèces prédites pour les SPB qui ont été échantillonnées dans les pièges à émergence (espèces «au rendez-vous ») ne sont quasiment pas associées aux tissus des plantes herbacées. La quasiabsence de ces espèces au stade larvaire dans les SPB pourrait s'expliquer non seulement par des fauches encore trop précoces, qui ne permettent pas à ces espèces d'effectuer la totalité de leur développement larvaire dans les tissus des végétaux, mais également par la sous-représentation de certaines espèces de plantes témoins d'une certaine forme d'artificialisation de ces SPB.

\subsection{Relations végétation-syrphes}

La végétation présente dans ces SPB est essentiellement décrite du point de vue des espèces qui les composent. Néanmoins, les caractéristiques fonctionnelles des espèces végétales semblent jouer un rôle essentiel visà-vis des insectes pollinisateurs (Landis et al., 2000 ; Uyttenbroeck et al., 2017 ; Gardarin et al., 2018).

Nous montrons que certaines caractéristiques fonctionnelles végétales sont en relation avec les syrphes qui exploitent ces surfaces au stade adulte. En effet, en plus du rôle que les SPB jouent en tant que milieu pour le développement larvaire des syrphes, elles constituent un milieu riche en ressources trophiques (nectar et pollen) pour les syrphes adultes provenant des SPB, mais aussi des milieux cultivés ou boisés périphériques. Nos données permettent d'établir deux relations statistiques positives: d'une part entre l'IE des SPB mesurée par les syrphes et la quantité de fleurs jaunes, d'autre part entre cette intégrité et la quantité de fleurs produisant du nectar. Ces relations sont établies de façons très globales puisque les syrphes sont échantillonnés par des pièges Malaise et non observés directement sur les fleurs. On sait ainsi que c'est l'accessibilité du nectar qui compte, plus que sa présence (Hatt et al., 2019). Concernant la couleur des fleurs, il est connu que certaines espèces possédant des fleurs jaunes fleurissent précocement et se maintiennent tardivement dans la saison. Elles fournissent donc aux espèces de syrphes précoces et plus tardives des ressources trophiques à un moment critique de la saison. Ranunculus acris subsp. friesianus, Rhinanthus alectorolophus, Taraxacum officinale aggr. et T.pratensis s.str. sont ainsi des espèces très fréquemment rencontrées dans les SPB qui fleurissent pour la plupart entre début avril et début mai et peuvent se maintenir en fleur jusque fin septembre. Ces espèces, n'ayant pas besoin d'une gestion particulièrement extensive pour se développer et ne faisant pas partie des espèces indicatrices permettant l'octroi du niveau de qualité II, sont souvent considérées comme «banales » du point de vue botanique. Elles paraissent donc importantes pour la faune syrphidologique.

La relation statistique mise en évidence entre la quantité de fleurs rose-violette et l'abondance en syrphes adultes est certainement à considérer avec prudence, puisque l'on considère que les syrphes ne se nourrissent généralement pas de fleurs de cette couleur (Speight, 2015), contredit par le cas du bleuet Centaurea cyanus dans Carrié et al. (2012). Il n'est pas exclu que cette relation (statistique) soit due à un même facteur externe influençant simultanément certaines plantes à fleurs rose-violette et les syrphes.

\section{CONCLUSIONS ET PERSPECTIVES}

La taille des SPB ainsi que leur qualité botanique (telle que définie dans la norme suisse) n'influencent pas la composition syrphidologique des prairies extensives étudiées. Ces résultats ont été obtenus autant à partir des syrphes émergents de ces surfaces et y ayant donc accompli leur développement, que des assemblages d'espèces interceptées en vol. La confrontation de ces deux cortèges constitue, à notre connaissance, un élément nouveau dans ce domaine.

Les résultats obtenus montrent que les SPB prairiales ont un potentiel de lutte biologique pouvant être bénéfique aux cultures périphériques. Pour que ces prairies apportent une réelle plus-value aux agriculteurs, la sélection d'espèces végétales indicatrices contenues dans les SPB pour l'obtention du niveau de qualité supérieure devrait essentiellement reposer sur des 
critères destinés à maintenir une diversité fonctionnelle permettant la lutte biologique par conservation. Il faudrait donc concevoir la composition des mélanges fleuris non pas uniquement sur la base de l'identité des espèces végétales qui les composent, mais y intégrer une dimension fonctionnelle visant à sélectionner les espèces végétales en fonction de caractéristiques essentielles aux interactions végétation-insectes auxiliaires, comme la couleur des fleurs ou leur capacité à produire nectar et pollen. Cette perspective devrait se fonder sur des études phénologiques plus fines des cortèges botaniques et syrphidologiques, ainsi que sur la mise en relation directe des espèces de syrphes et des ressources qu'elles utilisent, notamment à travers l'analyse des contenus stomacaux.

\section{Remerciements}

Nous remercions vivement toutes les personnes qui, de près ou de loin, se sont impliquées dans la réalisation de cette étude, en particulier Véronique Meyer et Philippe Roux de l'Office Cantonal de l'Agriculture et de la Nature de la République et du canton de Genève (OCAN), pour le partage des données relatives aux SPB et aux parcelles viticoles, les exploitants viticoles pour la mise à disposition de leurs parcelles, Aurélien Krause, civiliste à l'OCAN, Aurélia Passaseo, Patrick Charlier, Catherine Polli et Bernard Schaetti pour l'aide sur le terrain. Merci à l'institut des Sciences de l'Environnement (ISE) de l'Université de Genève pour le financement des pièges.

\section{Bibliographie}

Albrecht M.et al., 2007. The Swiss agri-environment scheme enhances pollinator diversity and plant reproductive. J. Appl. Ecol., 44, 813-822.

Almohamad R., Verheggen F. \& Haubruge É., 2009. Searching and oviposition behavior of aphidophagous hoverflies (Diptera: Syrphidae): a review. Biotechnol. Agron. Soc. Environ., 13(3), 467-481.

Bessat M., Delabays N., Castella E. \& Fleury D., 2018. Évaluation de la diversité végétale de douze prairies extensives classées en Surfaces de Promotion de la Biodiversité (SPB) à Genève. Saussurea, 47, 117-129.

Blaauw B.R. \& Isaacs R., 2014. Larger patches of diverse floral resources increase insect pollinator density, diversity, and their pollination of native wildflowers. Basic Appl.Ecol., 15(8), 701-711.

Burgio G. \& Sommaggio D., 2007. Syrphids as landscape bioindicators in Italian agroecosystems. Agric. Ecosyst. Environ., 120, 416-422.

Burgio G. et al., 2015. The influence of vegetation and landscape structural connectivity on butterflies (Lepidoptera: Papilionoidea and Hesperiidae), carabids (Coleoptera: Carabidae), syrphids (Diptera: Syrphidae), and sawflies (Hymenoptera: Symphyta) in Northern Italy farmland. Environ. Entomol., 44(5), 1300-1307.

Caillet-Bois D., Weiss B., Benz R. \& Stäheli B., 2016. Promotion de la biodiversité dans l'exploitation agricole: exigences de base et niveaux de qualité, conditions - charges - contributions. Lausanne, Suisse : Agridea.

Carrié R.J.G.， George D.R. ～\& Wäckers F.L., 2012. Selection of floral resources to optimise conservation of agriculturally-functional insect groups. J. Insect Conserv., 16(4), 635-640.

Chessel D., Dufour A.B. \& Thioulouse J., 2004. The ade4 package-I- One-table methods. $R$ News, 4, 5-10.

Cowgill S.E., Wratten S.D. \& Sotherton N.W., 1993. The selective use of floral resources by the hoverfly Episyrphus balteatus (Diptera: Syrphidae) on farmland. Ann. Appl. Biol., 122, 223-231.

Devillers P., Devillers-Terschuren J. \& Ledant J.-P., eds, 1991.CORINE biotopesmanual: habitats of the European Community, data specifications part 2. Luxembourg: Commission of the European Communities.

Ganser D., Knop E. \& Albrecht M., 2019. Sown wildflower strips as overwintering habitat for arthropods: effective measure or ecological trap? Agric. Ecosyst. Environ., 275, 123-131.

Gardarin A., Plantegenest M., Bischoff A. \& ValantinMorison M., 2018. Understanding plant-arthropod interactions in multitrophic communities to improve conservation biological control: useful traits and metrics. J. Pest Sci., 91(3), 943-955.

George D.R., Croft P., Northing P. \& Wäckers F.L., 2010. Perennial field margins with combined agronomical and ecological benefits for vegetable rotation schemes. BioControl, 56, 45-48.

Gilbert F.S., 1981. Foraging ecology of hoverflies: morphology of the mouthparts in relation to feeding on nectar and pollen in some common urban species. Ecol. Entomol., 10, 385-392.

Gurr G.M., Wratten S.D. \& Altieri M.A., 2004. Ecological engineering for pest management: 1-12, In: Gurr. G.M. et al. Ecological engineering for pest management advances in habitat manipulation for arthropods. Ithaca, NY, USA: Cornell University Press.

Haenke S., 2009. Increasing syrphid fly diversity and density in sown flower strips within simple vs. complex landscapes. J. Appl. Ecol., 46, 1106-1114.

Hajek A., 2004. Natural enemies: an introduction to biological control. Cambridge, UK: Cambridge University Press.

Hatt S. et al., 2017. Pest regulation and support of natural enemies in agriculture: experimental evidence of within field wildflower strips. Ecol. Eng., 98, 240-245.

Hatt S. et al., 2019. Identification of flower functional traits affecting abundance of generalist predators in perennial multiple species wildflower strips. Arthropod-Plant Interact., 13(1), 127-137. 
Kleijn D. \& Van Langevelde F., 2006. Interacting effects of landscape context and habitat quality on flower visiting insects in agricultural landscapes. Basic Appl. Ecol., 7, 201-214.

Kühn I., Durka W. \& Klotz S., 2004. BiolFlor, a planttrait database, http://www2.ufz.de/biolflor/overview/ merkmale.jsp, (20.07.2017).

Landis D.A., Wratten S.D. \& Gurr G.M., 2000. Habitat management to conserve natural enemies of arthropod pests in agriculture. Ann. Rev. Entomol., 45, 175-201.

Lazaro A., Hegland S.J. \& Totlannd O., 2008. The relationships between floral traits and specificity of pollination systems in three Scandinavian plant communities. Oecologia, 157, 249-257.

Londo G., 1976. The decimal scale for releves of permanent quadrats. Vegetatio, 33, 61-64.

Malaise R., 1937. A new insect-trap. Entomologisk Tidskrift, 58, 148-160.

Meyer B., Jauker F. \& Steffan-Dewenter I., 2009. Contrasting resource-dependent responses of hoverfly richness and density to landscape structure. Basic Appl. Ecol., 10, 178-186.

OCAN (Office Cantonal de l'Agriculture et de la Nature), 2019. Rapport d'activité 2019. Genève, Suisse : OCAN.

Oksanen J. et al., 2017. Package 'vegan', https:// cran.r-project.org/web/packages/vegan/vegan.pdf, $(13 / 09 / 2017)$.

Östman Ö., Ekbom B. \& Bengtsson J., 2001. Landscape heterogeneity and farming practice influence biological control. Basic Appl. Ecol., 2, 365-371.

Ouin A. et al., 2006. The species-area relationship in the hoverfly (Diptera, Syrphidae) communities of forest fragments in southern France. Ecography, 29, 183-190.

Ouvrard P., Transon J. \& Jacquemart A.L., 2018. Flowerstrip agri-environment schemes provide diverse and valuable summer flower resources for pollinating insects. Biodivers. Conserv., 27(9), 2193-2216.

R Development Core Team, 2008. R: A language and environment for statistical computing. Vienna: Austria R Foundation for Statistical Computing, http://www.Rproject.org, (01/10/2016).

Sarthou V. \& Speight M.C.D., 2013. Évaluation du potentiel de biodiversité à l'aide des diptères syrphides : le cas d'écosystèmes forestiers, zone du Mont Aigoual (Parc National des Cévennes, Lozère). Dublin : Syrph the Net Publications.

Schaffers A.P., Raemarkers I.P., Sykora V. \& Ter Braak C.J.F., 2008. Arthropod assemblages are best predicted by plant species composition. Ecology, 89(3), 782-794.

Sommaggio D., 1999. Syrphidae: can they be used as environmental bioindicators? Agric. Ecosyst. Environ., 74, 343-356.

Speight M.C.D., 2015. Species accounts of European syrphidae (Diptera). Dublin: Syrph the Net publications.

Speight M.C.D., Castella E. \& Obrdlik P., 2000. Use of the Syrph the Net database 2000. In: Speight M.C.D., Castella E., Obrdlik P. \& Ball S., eds. Syrph the Net, the database of European Syrphidae. Dublin: Syrph the Net publications.

Speight M.C.D. \& Castella E., 2001. An approach to interpretation of lists of insects using digitised biological information about the species. J. Insect Conserv., 5, 131139.

Speight M.C.D. \& Castella E., 2015. StN Database: content and glossary of terms, 2015. Dublin: Syrph the Net publications.

Speight M.C.D. et al., 2019. Révision de la liste des Diptères Syrphidae du canton de Genève, incluant cinq espèces nouvelles pour la Suisse. Entomo Helvetica, 12, 87-98.

Sutherland J.P., Sullivan M.S. \& Poppy G.M., 2001. Distribution and abundance of aphidophagous hoverflies (Diptera: Syrphidae) in wildflower patches and field margin habitats. Agric. For. Entomol., 3(1), 57-64.

Thies C., Steffan-Dewenter I. \& Tscharntke T., 2003. Effects of landscape context on herbivory and parasitism at different spatial scales. Oikos, 101, 18-25.

Tscharntke T. \& Brandl R., 2004. Plant-insect interactions in fragmented landscapes. Ann. Rev. Entomol., 49, 405430 .

Tscharntke T.. et al., 2005. Landscape perspectives on agricultural intensification and biodiversity - ecosystem service management. Ecol. Lett., 8, 857-874.

Uyttenbroeck R. et al., 2017. Increasing plant functional diversity is not the key for supporting pollinators in wildflower strips. Agric. Ecosyst. Environ., 249, 144155.

Van Veen M.P., 2004. Hoverflies of Northwest Europe: identification keys to the Syrphidae. Utrecht, The Netherlands: KNNV Publishing.

Zurbrügg C. \& Frank T., 2006. Factors influencing bug diversity (Insecta: Heteroptera) in semi-natural habitats. Biodivers. Conserv., 15, 275-294.

(48 réf.) 